\title{
Readmission following hypoxic ischemic brain injury: a population-based cohort study
}

\author{
Vincy Chan PhD, David Stock PhD, Binu Jacob MSc PhD, Nora Cullen MD, \\ Angela Colantonio PhD OT Reg. (Ont.)
}

\section{Abstract}

Background: Readmission to acute care is common and is associated with indicators of suboptimal care and health system inefficiencies. The objective of this study was to identify independent determinants of readmission following survival of hypoxic ischemic brain injury.

Methods: We conducted a population-based retrospective cohort study using Ontario's administrative health data. Survivors of hypoxic ischemic brain injury aged 20 years or more discharged from acute care between fiscal years 2002/03 and 2010/11 were included. Multivariable negative binomial regression was used to identify independent determinants of both number of readmissions and cumulative duration of hospital stay(s) within 1 year after the index discharge.

Results: Of the 593 patients with hypoxic ischemic brain injury, 233 (39.3\%) were readmitted within 1 year of the index acute care discharge. The number of readmissions was associated with age (35-49 yr v. 65-79 yr: rate ratio [RR] 0.57, 95\% confidence interval [Cl] 0.38-0.85; $\geq 80$ yr v. 65-79 yr: RR 0.58, 95\% Cl 0.34-0.97) and higher comorbidity score (Johns Hopkins Aggregated Diagnosis Groups score > 30 v. < 10: RR 1.60, 95\% Cl 1.11-2.31). Cumulative readmission stay was associated with increased index acute care length of stay (31-90 d v. $\geq 90 \mathrm{~d}$ : RR 4.17, 95\% Cl 1.38-12.64), prior use of health care services (minimal v. very high: RR 0.15, $95 \% \mathrm{Cl} 0.05-0.49$ ) and discharge disposition (home v. continuing/long-term care: RR 0.44, 95\% $\mathrm{Cl} 0.21-0.91$ ).

Interpretation: The findings indicate a high readmission rate in the first year after the index acute care admission for survivors of hypoxic ischemic brain injury, reflecting care gaps and system inefficiencies. This suggests that bolstered discharge and home care planning and support are needed to address the specific needs of those with hypoxic ischemic brain injury.

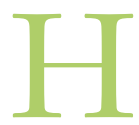

ypoxic ischemic brain injury is a nontraumatic brain injury that results from sustained oxygen deprivation arising from ischemic (i.e., restricted blood flow) or anoxic/hypoxic (i.e., no/low oxygen irrespective of ischemia) origins. ${ }^{1,2}$ The common causes are cardiac or respiratory arrest, near drowning, carbon monoxide poisoning and asphyxia. ${ }^{3,4}$ Patients with hypoxic ischemic brain injury place a considerable burden on the health care system, with about 1 in 2 patients experiencing at least 1 alternate level of care day (days in which acute medical care is no longer required yet the patient still occupies an acute care bed $\left.{ }^{5}\right){ }^{6}$ a negative system-level outcome that is costly for the health care system and for patients waiting for appropriate care.

Readmission to hospital is an equally negative system-level outcome that is costly to both the patient and the health care system. ${ }^{7.8}$ Between 2010 and 2011, 8.5\% of Canadian patients were readmitted to hospital within 30 days, resulting in an estimated associated cost of $\$ 1.8$ billion to the health care system. ${ }^{9}$ Readmission rates among patients with a traumatic brain injury (TBI) range from $16 \%$ to $29 \%,{ }^{10-14}$ with acute care costs accounting for $46 \%-65 \%$ of care expenditures during the year after the injury. ${ }^{15}$ The aggregate first-year medical costs for patients with TBI and those with nontraumatic brain injury were estimated at $\$ 120.7$ million and $\$ 368.7$ million, respectively, within the Ontario-wide population discharged between 2004 and 2007. ${ }^{15}$ Factors associated with readmission to hospital among patients with TBI were age, injury severity, injury while on active duty, more comorbidities, previous acute care stays, inpatient rehabilitation length of stay and functional status on acute care discharge..$^{14,16-19}$ Discharge to

\section{Competing interests: None declared.}

This article has been peer reviewed.

Correspondence to: Vincy Chan, Vincy.Chan@uhn.ca

CMAJ Open 2018. DOI:10.9778/cmajo.20180080 
extended care facilities and inpatient rehabilitation facilities was also associated with 30-day readmission among older patients. ${ }^{20}$

Most research on readmission for brain-injury-related events has focused on the TBI population, with many bestpractice guidelines for the hypoxic ischemic brain injury population modelled after those set for TBI. This is problematic, as the former is distinct and differs significantly in terms of the demographic, clinical and environmental profile. ${ }^{21}$ Moreover, patients with hypoxic ischemic brain injury experience less functional improvement ${ }^{22,23}$ and greater psychosocial problems ${ }^{23}$ in the long term than patients with TBI. It is currently unknown whether readmission rates and factors associated with readmission identified among the TBI population are common to those with hypoxic ischemic brain injury. As such, planning and prevention of readmission for hypoxic ischemic brain injury using these data are vague, and there is a need for evidence on determinants of acute care readmission specific to this population. The objective of this study was to address this gap by identifying factors that determine the number and cumulative duration of acute care readmissions among survivors of hypoxic ischemic brain injury. Understanding which factors determine readmission can inform policy aimed at minimizing avoidable readmission and thereby support effective health planning specific to hypoxic ischemic brain injury for Canadian survivors of these injuries.

\section{Methods}

\section{Study design}

We conducted a retrospective cohort study to analyze rates of readmission to hospital within 1 year of acute care discharge among patients with hypoxic ischemic brain injury. Population-based administrative data on acute care were extracted from the Canadian Institute for Health Information Discharge Abstract Database (DAD), which contains demographic and clinical information on all hospital discharges and deaths from all publicly funded hospitals in Ontario. Data in the DAD were obtained through the Ontario Cancer Data Linkage Project (cd-link), an initiative of the Ontario Institute for Cancer Research/Cancer Care Ontario Health Services Research Program whereby risk-reduced coded data from the Institute for Clinical Evaluative Sciences are provided directly to researchers, with the protection of a comprehensive data use agreement.

\section{Setting and patients}

Adult patients (age $\geq 20 \mathrm{yr}$ ) with hypoxic ischemic brain injury who had been discharged from acute care between fiscal years 2002/03 and 2010/11 were identified from the DAD. Inclusion of hypoxic ischemic brain injury discharges stopped at Mar. 31, 2011 to allow us to follow these patients for up to 1 year in our data set to identify readmissions. We identified patients using International Statistical Classification of Diseases and Related Health Problems, 10th revision, enhanced Canadian version (ICD-10-CA) diagnostic codes. There is currently no consensus on the case definition for hypoxic ischemic brain injury. To compensate for the absence of an empirically validated clinical case definition or formal diagnostic criteria for the condition, we used the broader definition of anoxic brain damage as the most responsible diagnosis, or probable causes of hypoxic ischemic brain injury, including toxic encephalopathy and toxic effect of carbon monoxide, as the most responsible diagnosis, coincident with the ICD10-CA code for anoxic brain damage (G93.1) as a secondary acute care diagnosis. ${ }^{5,18,24}$ Thus, the following patients were included: those with a most responsible diagnosis code of G93.1 and those with codes for most responsible diagnosis that corresponded to probable causes of hypoxic ischemic brain injury (I46.0 [cardiac arrest with successful resuscitation], R09.0 [asphyxia], R09.2 [respiratory arrest], T71 [asphyxiation], T75.1 [drowning and nonfatal submersion], G92 [toxic encephalopathy], T58 [toxic effect of carbon monoxide] or T70.2 [other and unspecified effects of high altitude]), with G93.1 in any secondary diagnostic field.

\section{Outcomes}

The outcomes of interest were 1) the number of acute care readmissions following discharge after the index acute care episode for hypoxic ischemic brain injury and 2) the cumulative duration of hospital stays within 1 year (defined as 4-365 d) after the index acute care discharge.

\section{Potential determinants of readmission}

We assessed potential demographic, clinical and acute care episode determinants of readmission within 1 year. The demographic variables included age at admission, sex, neighbourhood income quintile and rurality, the latter 2 aggregated to the level of dissemination area, the smallest divisible geographical Canadian census unit. The clinical and acute care episode characteristics included fiscal year of acute care admission, number of special care hours, index acute care length of stay, number of alternate level of care days during the index acute care episode, presence of 1 or more psychological/behavioural conditions (identified by a Chapter 5 ICD-10-CA code in any of the index admission diagnostic fields) and index acute care discharge disposition. We derived a comorbidity index from a weighted sum of the Johns Hopkins Aggregated Diagnosis Groups accumulated over the 2 years before the acute care episode; the index measure has been validated with Ontario administrative health data. ${ }^{25} \mathrm{We}$ measured use of health care services using the Johns Hopkins Adjusted Clinical Groups Resource Utilization Bands category. ${ }^{26,27}$

\section{Statistical analysis}

We used negative binomial count regression models to determine the independent relative effects of covariates on the number of readmissions and the duration of these hospital stays. Owing to overdispersion (Pearson $\chi^{2}$ test), we did not use a Poisson distribution. Visual inspection of predicted versus relative outcome frequencies indicated negligibly improved fit with a zero-inflated model. We examined multiple individual models comprising each potential covariate and 
the outcome variable. We used forward selection, where each potential determinant was entered in order of most significant (based on the likelihood ratio test from bivariable models) to least significant. Age and sex were forced into all models. Potential determinants were not retained if the $p$ value was $>0.1$. Given the large proportion of patients who died in the year after the index acute care episode, we used an offset variable to adjust person-time contribution.

\section{Ethics approval}

The study was approved by the Research Ethics Board of the University Health Network, Toronto.

\section{Results}

Between 2002/03 and 2010/11, there were 2880 patients with an acute care visit related to a hypoxic ischemic brain injury, of whom $2112(73.3 \%)$ had ICD-10-CA code G93.1 as the most responsible diagnosis. Of the 593 patients who survived their index acute care episode, 233 (39.3\%) were readmitted to acute care within 1 year of discharge, some more than once (average number of readmissions 1.79 [standard deviation 1.31]), resulting in 417 readmissions. Of the 593 patients, 174 $(29.3 \%)$ died within 1 year of discharge. These patients accounted for 4758 inpatient hospital days (average duration of stay 20.42 d [standard deviation $39.21 \mathrm{~d}$ ]) within 1 year of the index acute care discharge. Patients with anoxic brain damage and those with "awaiting admission to adequate facility elsewhere" (ICD-10-CA code Z75.1) as the most responsible diagnosis accounted for $13.6 \%$ and $8.2 \%$, respectively, of readmission days. Of the 233 patients readmitted at least once, 108 (46.4\%) were aged 65 years or more, 108 (46.4\%) were men, $207(88.8 \%)$ had moderate to very high Adjusted Clinical Groups Resource Utilization Bands, 63 (27.0\%) had more than 30 Aggregated Diagnosis Groups comorbidities, $171(73.4 \%)$ had an index acute care stay of 10-90 days, and $63(27.0 \%)$ were discharged home after the index acute care event (Table 1$)$. Seventeen patients $(2.9 \%)$ had stroke as a secondary diagnosis; additional analyses showed that excluding these 17 patients did not materially affect estimated effects on number of readmissions or duration of hospital stay(s) within 1 year.

Relative effects of potential determinants on the number of readmissions and duration of acute care stay(s) within 1 year after the index acute care discharge are presented in Table 2. The rate of readmission among patients aged 35-49 years (rate ratio $[\mathrm{RR}]$ 0.57, 95\% confidence interval [CI] 0.38-0.85) and 80 years or more (RR $0.58,95 \%$ CI $0.34-0.97$ ) was more than $40 \%$ lower than the rate for patients aged $65-79$ years. A reduced readmission rate was also observed for patients aged 50-64 years (RR 0.72, 95\% CI 0.52-1.00), although this was of borderline statistical significance. An index acute care episode Aggregated Diagnosis Groups score of more than 30 was associated with a $60 \%$ (RR $1.60,95 \%$ CI 1.11-2.31) increased rate of readmission relative to a score of less than 10 . There was suggestion of an increased likelihood of readmission with increasing amount of time spent in the special care unit, being free of a psychological/behavioural diagnosis, or having been discharged home or to inpatient rehabilitation instead of continuing/long-term care, although the point estimates were not statistically significant.

Compared to patients whose index acute care stay was 90 days or more, those with an index stay of 10-30 days and those with an index stay of 31-90 days were likely to spend 2.88 (95\% CI 1.28-6.52) and 4.17 (95\% CI 1.38-12.64) more days, respectively, in hospital within a year following discharge. Those who used minimal health care services within the prior 2 years (Adjusted Clinical Groups Resource Utilization Bands category healthy/none) were likely to spend 0.15 fold fewer days (RR 0.15, 95\% CI 0.05-0.49) in hospital during the year after the index acute care episode relative to patients whose prior use of services was very high. Compared to patients discharged to continuing/long-term care from their index acute care episode, those discharged home were likely to spend fewer days in an acute care bed during the following year (RR 0.44, 95\% CI 0.21-0.91). The interaction between age and sex was not significant in both models.

\section{Interpretation}

In this study of determinants of hospital readmission specific to patients with hypoxic ischemic brain injury, our findings suggest that the proportion of patients readmitted within 1 year of the index acute care discharge, 39.3\%, was higher than that reported for patients with TBI $(16 \%-29 \%) .{ }^{12-14}$ Observed determinants of fewer readmissions were both older and younger age relative to patients aged 65-79 years, whereas increased prior comorbidity was associated with more readmissions. Observed determinants of the cumulative duration of the acute care stay(s) in the year after the index acute care discharge were having minimal prior contact with the health care system, an index episode length of stay of 10-90 days and being discharged to continuing/long-term care instead of home after the index acute care episode.

These findings are similar to those identified for patients with TBI, medical and surgical patients, and all-cause readmissions. ${ }^{28}$ It is plausible that patients aged 35-64 years are less likely be readmitted after discharge from an acute care stay for hypoxic ischemic brain injury because they are less frail than the reference group of those aged 65-79 years. The oldest patients who survive their acute care episode may be less likely to be readmitted chiefly owing to having less severe hypoxic ischemic brain injuries, as almost $80 \%$ of patients with hypoxic ischemic brain injury admitted to acute care die in hospital. ${ }^{5}$ Socioeconomic status indicators such as neighbourhood income quintile and rurality were not associated with readmission in the current study, a finding consistent with the Canadian Institute for Health Information data on all-cause readmission. ${ }^{9}$

We found that discharge home instead of to continuing/ long-term care from the index acute care episode was associated with a greater number of readmissions but fewer days in acute care over the year. Studies have shown that discharge destination other than home is independently associated with 


\begin{tabular}{|c|c|c|c|c|}
\hline \multirow[b]{2}{*}{ Characteristic } & \multirow{2}{*}{$\begin{array}{c}\text { No. }(\%) \text { of } \\
\text { discharges } \\
n=593\end{array}$} & \multicolumn{2}{|c|}{$\begin{array}{l}\text { Readmission within } 1 \mathrm{yr} \\
\text { no. }(\%) \text { of patients }\end{array}$} & \multirow[b]{2}{*}{$p$ value } \\
\hline & & $\begin{array}{c}\text { Yes } \\
n=233\end{array}$ & $\begin{array}{c}\text { No } \\
n=360\end{array}$ & \\
\hline \multicolumn{5}{|l|}{ Age at admission, yr } \\
\hline 20-34 & $54(9.1)$ & $20(8.6)$ & $34(9.4)$ & 0.2 \\
\hline $35-49$ & $107(18.0)$ & $35(15.0)$ & $72(20.0)$ & \\
\hline $50-64$ & $189(31.9)$ & $70(30.0)$ & $119(33.0)$ & \\
\hline $65-79$ & $188(31.7)$ & $85(36.5)$ & $103(28.6)$ & \\
\hline$\geq 80$ & $55(9.3)$ & $23(9.9)$ & $32(8.9)$ & \\
\hline \multicolumn{5}{|l|}{ Sex } \\
\hline Female & $204(34.4)$ & $72(30.9)$ & $132(36.7)$ & 0.1 \\
\hline Male & $389(65.6)$ & $161(69.1)$ & $228(63.3)$ & \\
\hline \multicolumn{5}{|l|}{ Income quintile } \\
\hline 1 (lowest) & $165(27.8)$ & $62(26.6)$ & $103(28.6)$ & 0.8 \\
\hline 2 & $126(21.2)$ & 52 (22.3) & $74(20.6)$ & \\
\hline 3 & $105(17.7)$ & $42(18.0)$ & $63(17.5)$ & \\
\hline 4 & $123(20.7)$ & $52(22.3)$ & $71(19.7)$ & \\
\hline 5 (highest) & $74(12.5)$ & $25(10.7)$ & $49(13.6)$ & \\
\hline \multicolumn{5}{|l|}{ Rurality } \\
\hline Rural & $58(9.8)$ & $23(9.9)$ & $35(9.7)$ & 1.0 \\
\hline Urban & $535(90.2)$ & $210(90.1)$ & $325(90.3)$ & \\
\hline \multicolumn{5}{|c|}{$\begin{array}{l}\text { Johns Hopkins ACG } \\
\text { Resource Utilization Bands } \\
\text { category }\end{array}$} \\
\hline None or healthy & $34(5.7)$ & $13(5.6)$ & $21(5.8)$ & 0.7 \\
\hline Low & $35(5.9)$ & $13(5.6)$ & $22(6.1)$ & \\
\hline Moderate & $198(33.4)$ & 75 (32.2) & $123(34.2)$ & \\
\hline High & $152(25.6)$ & $56(24.0)$ & $96(26.7)$ & \\
\hline Very high & $174(29.3)$ & $76(32.6)$ & $98(27.2)$ & \\
\hline \multicolumn{5}{|l|}{ Comorbidity index ${ }^{*}$} \\
\hline$<10$ & $187(31.5)$ & $69(29.6)$ & $118(32.8)$ & 0.3 \\
\hline $10-30$ & $265(44.7)$ & $101(43.3)$ & $164(45.6)$ & \\
\hline$>30$ & $141(23.8)$ & $63(27.0)$ & $78(21.7)$ & \\
\hline \multicolumn{5}{|l|}{ Fiscal year } \\
\hline 2002/03-2003/04 & $137(23.1)$ & $50(21.4)$ & $87(24.2)$ & 0.9 \\
\hline 2004/05-2005/06 & $123(20.7)$ & $51(21.9)$ & $72(20.0)$ & \\
\hline $2006 / 07-2007 / 08$ & $131(22.1)$ & $50(21.4)$ & $81(22.5)$ & \\
\hline 2008/09-2009/10 & $138(23.3)$ & $56(24.0)$ & $82(22.8)$ & \\
\hline $2010 / 11$ & $64(10.8)$ & 26 (11.2) & $38(10.6)$ & \\
\hline \multicolumn{5}{|c|}{ No. of special care hours } \\
\hline 0 & $86(14.5)$ & $31(13.3)$ & 55 (15.3) & 0.6 \\
\hline $1-499$ & $333(56.2)$ & $127(54.5)$ & $206(57.2)$ & \\
\hline 500-999 & $95(16.0)$ & 40 (17.2) & 55 (15.3) & \\
\hline$\geq 1000$ & 79 (13.3) & $35(15.0)$ & $44(12.2)$ & \\
\hline
\end{tabular}




\begin{tabular}{|c|c|c|c|c|}
\hline \multirow[b]{2}{*}{ Characteristic } & \multirow{2}{*}{$\begin{array}{c}\text { No. }(\%) \text { of } \\
\text { discharges } \\
n=593\end{array}$} & \multicolumn{2}{|c|}{$\begin{array}{l}\text { Readmission within } 1 \mathrm{yr}, \\
\text { no. }(\%) \text { of patients }\end{array}$} & \multirow[b]{2}{*}{$p$ value } \\
\hline & & $\begin{array}{c}\text { Yes } \\
n=233\end{array}$ & $\begin{array}{c}\text { No } \\
n=360\end{array}$ & \\
\hline \multicolumn{5}{|l|}{ Index length of stay, $d$} \\
\hline$<10$ & $119(20.1)$ & $37(15.9)$ & $82(22.8)$ & 0.1 \\
\hline $10-30$ & $203(34.2)$ & $77(33.0)$ & $126(35.0)$ & \\
\hline $31-90$ & $210(35.4)$ & $94(40.3)$ & $116(32.2)$ & \\
\hline$>90$ & $61(10.3)$ & $25(10.7)$ & $36(10.0)$ & \\
\hline \multicolumn{5}{|c|}{$\begin{array}{l}\text { No. of alternate level of care } \\
\text { days }\end{array}$} \\
\hline 0 & $354(59.7)$ & $131(56.2)$ & $223(61.9)$ & 0.4 \\
\hline $1-14$ & $100(16.9)$ & $44(18.9)$ & $56(15.6)$ & \\
\hline$>14$ & $139(23.4)$ & $58(24.9)$ & $81(22.5)$ & \\
\hline \multicolumn{5}{|l|}{$\begin{array}{l}\text { Psychological/behavioural } \\
\text { diagnosis }\end{array}$} \\
\hline Absent & $452(76.2)$ & $185(79.4)$ & $267(74.2)$ & 0.1 \\
\hline Present & $141(23.8)$ & $48(20.6)$ & $93(25.8)$ & \\
\hline \multicolumn{5}{|l|}{ Discharge disposition } \\
\hline Home & $130(21.9)$ & $63(27.0)$ & $67(18.6)$ & 0.02 \\
\hline Rehabilitation & $122(20.6)$ & $54(23.2)$ & $68(18.9)$ & \\
\hline $\begin{array}{l}\text { Continuing/long-term } \\
\text { care }\end{array}$ & $181(30.5)$ & $62(26.6)$ & $119(33.0)$ & \\
\hline Other & $160(27.0)$ & $54(23.2)$ & $106(29.4)$ & \\
\hline $\begin{array}{l}\text { Note: } A C G=\text { Adjusted Clinical } \\
{ }^{*} \text { Derived from a weighted sum } \\
\text { the acute care admission. }\end{array}$ & $\begin{array}{l}=\text { Aggregated } \\
\text { topkins Aggre }\end{array}$ & $\begin{array}{l}\text { Groups. } \\
\text { nosis Groups }\end{array}$ & mulated over th & ears before \\
\hline
\end{tabular}

increased rate of readmission among patients with TBI and other trauma populations ${ }^{20,28}$ and is likely an indicator of medical complexity and injury severity. Readmission among patients with TBI has been suggested to arise from postdischarge care that is ill-equipped to resolve sudden complications, and prevention protocol planning for such events has been recommended..$^{29}$ Our finding also suggests that this group of survivors of hypoxic ischemic brain injury may, on average, have been lacking caregiver support at home, leading to more readmissions. However, overall, they may have had less complex conditions owing to a less severe hypoxic ischemic brain injury or comorbidity profile, therefore spending fewer days in the acute care setting when readmitted.

There is growing evidence that the care of patients with complex conditions can benefit substantially from interdisciplinary-team-based models. These can facilitate more optimal management of comorbid health conditions during acute care as well as improved coordination of discharge planning to ensure the necessary formal and informal supports are in place. ${ }^{30}$ Strategies including the introduction of new subacute care facilities, auxiliary nurses to support specialist nursing staff and summaries of specialist consultations retained in the patient's home may prevent inappropriate admissions or reduce length of stay, ${ }^{31}$ thus avoiding the decline into frailty characteristic of patients who linger unnecessarily in hospital. ${ }^{32}$ Adequate planning and support services during the transition from inpatient and/or rehabilitation facilitates to home and afterward are crucial in preventing the exacerbation of care needs, manageable in the current setting, into those requiring readmission. Transition supports emphasizing a more holistic approach to patient care both before and after discharge and available home care have been shown to maximize functional independence and overall health and to directly prevent readmission. ${ }^{33,34}$ Health care professionals and service organizations should factor in individual need in the context of available family and social supports, as well as be aware of ongoing health care and rehabilitation needs to facilitate access to required services. These processes require coordination between multiple health care, social and community support systems.

\section{Limitations}

Limitations associated with the use of health administrative data are recognized. The data captured in the DAD include 


\section{Table 2: Rate ratios for potential determinants of number of readmissions and cumulative duration of acute care stay(s) within 1 year of index acute care discharge}

\begin{tabular}{|c|c|c|}
\hline \multirow[b]{2}{*}{ Potential determinant } & \multicolumn{2}{|c|}{ Outcome; RR (95\% Cl) } \\
\hline & $\begin{array}{l}\text { No. of } \\
\text { readmissions }\end{array}$ & $\begin{array}{c}\text { Cumulative } \\
\text { duration of stay(s) }\end{array}$ \\
\hline \multicolumn{3}{|l|}{ Age at admission, yr } \\
\hline $20-34$ & $0.93(0.57-1.51)$ & $0.64(0.25-1.67)$ \\
\hline $35-49$ & $0.57(0.38-0.85)$ & $0.51(0.23-1.09)$ \\
\hline $50-64$ & $0.72(0.52-1.00)$ & $0.71(0.37-1.39)$ \\
\hline $65-79$ & 1.00 & 1.00 \\
\hline$\geq 80$ & $0.58(0.34-0.97)$ & $0.70(0.27-1.80)$ \\
\hline \multicolumn{3}{|l|}{ Sex } \\
\hline Female & $1.06(0.80-1.40)$ & $1.03(0.57-1.86)$ \\
\hline Male & 1.00 & 1.00 \\
\hline $\begin{array}{l}\text { Johns Hopkins ACG } \\
\text { Resource Utilization } \\
\text { Bands category }\end{array}$ & NR & \\
\hline None or healthy & & $0.15(0.05-0.49)$ \\
\hline Low & & $0.85(0.24-3.02)$ \\
\hline Moderate & & $0.68(0.36-1.28)$ \\
\hline High & & $0.71(0.37-1.40)$ \\
\hline Very high & & 1.00 \\
\hline Comorbidity index & & NR \\
\hline$<10$ & 1.00 & \\
\hline $10-30$ & $1.19(0.85-1.65)$ & \\
\hline$>30$ & $1.60(1.11-2.31)$ & \\
\hline Index length of stay, d & NR & \\
\hline$<10$ & & $1.67(0.78-3.59)$ \\
\hline $10-30$ & & $2.88(1.28-6.52)$ \\
\hline $31-90$ & & $4.17(1.38-12.64)$ \\
\hline$>90$ & & 1.00 \\
\hline No. of special care hours & & NR \\
\hline 0 & $0.88(0.58-1.33)$ & \\
\hline $1-499$ & 1.00 & \\
\hline 500-999 & $1.22(0.85-1.77)$ & \\
\hline$\geq 1000$ & $1.47(0.98-2.19)$ & \\
\hline $\begin{array}{l}\text { Psychological/ } \\
\text { behavioural diagnosis }\end{array}$ & & NR \\
\hline Absent & 1.00 & \\
\hline Present & $0.73(0.52-1.01)$ & \\
\hline \multicolumn{3}{|l|}{ Discharge disposition } \\
\hline Home & $1.41(0.97-2.06)$ & $0.44(0.21-0.91)$ \\
\hline Rehabilitation & $1.43(0.97-2.09)$ & $1.25(0.60-2.63)$ \\
\hline $\begin{array}{l}\text { Continuing/long-term } \\
\text { care }\end{array}$ & 1.00 & 1.00 \\
\hline Other & $1.09(0.74-1.60)$ & $1.73(0.74-4.06)$ \\
\hline
\end{tabular}

Note: $A C G=$ Adjusted Clinical Groups, $A D G=$ Aggregated Diagnosis Groups, $\mathrm{Cl}=$ confidence interval, $\mathrm{NR}=$ not retained in the model, $\mathrm{RR}=$ rate ratio all acute care discharges for Ontario. In the absence of population-wide clinical data specific for hypoxic ischemic brain injury, the $\mathrm{DAD}$ provides the most complete data coverage available for understanding readmissions among these patients at a population level. Furthermore, recruiting a study sample of similar magnitude for primary data collection would be unfeasible. Information on many of the factors that contribute to readmission, such as injury severity, functional independence at acute care discharge, social support and availability of private insurance, is not available in Ontario hospital discharge administrative data. There is currently no consensus on the case definition for hypoxic ischemic brain injury, and milder cases may be missed in health administrative data, resulting in underreporting of patients with the condition. To overcome this lack of empirically validated clinical case definition or formal diagnostic criteria for hypoxic ischemic brain injury, we used a broader extended definition by including patients with a diagnosis consistent with causes of hypoxic ischemic brain injury as a main diagnosis, with ICD-10-CA codes for anoxic brain damage as a secondary diagnosis. Our team used this algorithm previously. ${ }^{5,18,24}$ We included patients with toxic encephalopathy and toxic effect of carbon monoxide as the most responsible diagnosis coincident with anoxic brain damage as a secondary diagnosis in our case definition based on clinical observations of similarity in recovery trajectory. We were unable to conduct sensitivity analyses isolating the effect of toxic encephalopathy and of carbon monoxide because of small numbers, but we plan to investigate the effects of differences in injury pathology on recovery from hypoxic ischemic brain injury in future work. Similarly, while recognizing that determinants of readmission may differ by cause of injury and injury severity, we were unable to stratify analyses by these factors owing to limited study power. Although it is informative to adjust for the types of hypoxic ischemic brain injury, there is currently no information on the validity of using the secondary diagnostic codes in the data set to indicate the types of injury. Therefore, cause of hypoxic ischemic brain injury was not taken into account in our model. Although examining the effects of individual comorbidities would have been interesting, the study power for these analyses was limited. Instead, we used a parsimonious approach of adjusting for overall comorbidity burden using an index measure derived from the Johns Hopkins Aggregated Diagnosis Groups case-mix system. Furthermore, a quality assessment of DAD indicated near-perfect agreement for nonclinical variables and moderate to substantial agreement for the most responsible diagnosis. ${ }^{35}$ Finally, findings from this Ontario-wide study represent a substantial proportion (39\%) of the Canadian population and may be generalizable to jurisdictions with comparable acute care systems and postdischarge formal and informal support structures.

\section{Conclusion}

Our results suggest that more than 1 of every 3 patients with hypoxic ischemic brain injury discharged from hospital between 2002/03 and 2010/11 in Ontario was readmitted within 1 year. The findings support the literature on other 
brain-injured populations that interdisciplinary teams in the inpatient setting for patients with brain injury would be beneficial and highlight an unmet need for appropriate and adequate support in the transition from acute care to home. This includes the provision of home care services to ensure that patients are adequately cared for after discharge. The results also highlight the need for research specific to hypoxic ischemic brain injury to support evidence-based best practice in meeting the needs of these unique patients. Findings from this study will help inform health care service planning for this understudied population, thereby preventing avoidable care gaps and health system inefficiencies of which readmission rates are an indicator.

\section{References}

1. Howard RS, Holmes PA, Koutroumanidis MA. Hypoxic-ischaemic brain injury. Pract Neurol 2011;11:4-18.

2. Arciniegas DB. Hypoxic-ischemic brain injury: addressing the disconnect between pathophysiology and public policy. NeuroRehabilitation 2010;26:1-4

3. Lim C, Alexander MP, LaFleche G, et al. The neurological and cognitive sequelae of cardiac arrest. Neurology 2004;63:1774-8.

4. Busl KM, Greer DM. Hypoxic-ischemic brain injury: pathophysiology, neuropathology and mechanisms. NeuroRehabilitation 2010;26:5-13

5. Amy C, Zagorski B, Chan V, et al. Acute care alternate-level-of-care days due to delayed discharge for traumatic and non-traumatic brain injuries. Healthc Policy 2012;7:41-55.

6. Stock D, Cowie C, Chan V, et al. Determinants of alternate-level-of-care delayed discharge among acute care survivors of hypoxic-ischemic brain injury: a population-based cohort study. CMA7 Open 2016;4:E689-97.

7. Jencks SF, Williams MV, Coleman EA. Rehospitalizations among patients in the Medicare fee-for-service program. N Engl 7 Med 2009;360:1418-28.

8. Anderson GF, Steinberg EP. Hospital readmissions in the Medicare population. NEngl 7 Med 1984;311:1349-53.

9. All-cause readmission to acute care and return to the emergency department. Ottawa: Canadian Institute for Health Information; 2012. Available: https://secure.cihi. $\mathrm{ca} /$ free_products/Readmission_to_acutecare_en.pdf (accessed 2018 May 30).

10. Intiso D, Fontana A, Maruzzi G, et al. Readmission to the acute care unit and functional outcomes in patients with severe brain injury during rehabilitation. Eur 7 Phys Rebabil Med 2017;53:268-76.

11. Nakase-Richardson R, Tran J, Cifu D, et al. Do rehospitalization rates differ among injury severity levels in the NIDRR Traumatic Brain Injury Model Systems program? Arch Phys Med Rehabil 2013;94:1884-90.

12. Marwitz JH, Cifu DX, Englander J, et al. A multi-center analysis of rehospitalizations five years after brain injury. 7 Head Trauma Rebabil 2001;16:307-17.

13. Cifu DX, Kreutzer JS, Marwitz JH, et al. Etiology and incidence of rehospitalization after traumatic brain injury: a multicenter analysis. Arch Phys Med Rebabil 1999;80:85-90.

14. Saverino C, Swaine B, Jaglal S, et al. Rehospitalization after traumatic brain injury: a population-based study. Arch Phys Med Rehabil 2016;97(Suppl 2):S19-25.

15. Chen A, Bushmeneva K, Zagorski B, et al. Direct cost associated with acquired brain injury in Ontario. BMC Neurol 2012;12:76.

16. Tran J, Hammond F, Dams-O'Connor K, et al. Rehospitalization in the first year following veteran and service member TBI: a VA TBI Model Systems Study. 7 Head Trauma Rehabil 2017;32:264-70.

17. Pretz CR, Graham JE, Middleton A, et al. Longitudinal investigation of rehospitalization patterns in spinal cord injury and traumatic brain injury among Medicare beneficiaries. Arch Phys Med Rehabil 2017;98:997-1003.

18. Chan V, Zagorski B, Parsons D, et al. Older adults with acquired brain injury: a population-based study. BMC Geriatr 2013;13:97.

19. Colantonio A, Croxford R, Farooq S, et al. Trends in hospitalization associated with traumatic brain injury in a publicly insured population, 1992-2002. 7 Trauma 2009;66:179-83.

20. Strosberg DS, Housley BC, Vazquez D, et al. Discharge destination and readmission rates in older trauma patients. F Surg Res 2017;207:27-32.

21. Colantonio A, Gerber G, Bayley M, et al. Differential profiles for patients with traumatic and non-traumatic brain injury. 7 Rehabil Med 2011:43:311-5.

22. Cullen NK, Park YG, Bayley MT. Functional recovery following traumatic vs non-traumatic brain injury: a case-controlled study. Brain Inj 2008;22:1013-20.

23. Harbinson M, Zarshenas S, Cullen NK. Long-term functional and psychosocial outcomes after hypoxic-ischemic brain injury: a case-controlled comparison to traumatic brain injury. PM R 2017;9:1200-7.
24. Stock D, Cowie C, Chan V, et al. Determinants of admission to inpatient rehabilitation among acute care survivors of hypoxic-ischemic brain injury: a prospective population-wide cohort study. Arch Phys Med Rehabil 2016;97:885-91.

25. Austin PC, van Walraven C, Wodchis WP, et al. Using the Johns Hopkins Aggregated Diagnosis Groups (ADGs) to predict mortality in a general adult population cohort in Ontario, Canada. Med Care 2011;49:932-9.

26. Zielinski A, Kronogård M, Lenhoff H, et al. Validation of ACG case-mix for equitable resource allocation in Swedish primary health care. BMC Public Health 2009;9:347.

27. Brilleman SL, Salisbury C. Comparing measures of multimorbidity to predict outcomes in primary care: a cross sectional study. Fam Pract 2013;30:172-8.

28. Moore L, Stelfox HT, Turgeon AF, et al. Rates, patterns, and determinants of unplanned readmission after traumatic injury: a multicenter cohort study. Ann Surg 2014;259:374-80.

29. Hammond FM, Horn SD, Smout RJ, et al. Readmission to an acute care hospital during inpatient rehabilitation for traumatic brain injury. Arch Phys Med Rehabil 2015;96(Suppl 8):S293-303.e1.

30. O'Leary KJ, Sehgal NL, Terrell G, et al.; High Performance Teams and the Hospital of the Future Project Team. Interdisciplinary teamwork in hospitals: a review and practical recommendations for improvement. 7 Hosp Med 2012;7: 48-54.

31. Hammond CL, Pinnington LL, Phillips MF. A qualitative examination of inappropriate hospital admissions and lengths of stay. BMC Health Serv Res 2009;9:44

32. Sutherland JM, Crump RT. Alternative level of care: Canada's hospital beds, the evidence and options. Healthc Policy 2013;9:26-34.

33. Courtney MD, Edwards HE, Chang AM, et al. Improved functional ability and independence in activities of daily living for older adults at high risk of hospital readmission: a randomized controlled trial. F Eval Clin Pract 2012;18: 128-34.

34. Tinetti ME, Charpentier P, Gottschalk M, et al. Effect of a restorative model of posthospital home care on hospital readmissions. 7 Am Geriatr Soc 2012;60: 1521-6.

35. Juurlink D, Preyra C, Croxford R, et al. Canadian Institute for Health Information Discharge Abstract Database: a validation study. Toronto: Institute for Clinical Evaluative Sciences; 2006.

Affiliations: Toronto Rehabilitation Institute (Chan, Stock, Jacob, Cullen, Colantonio), University Health Network, Toronto, Ont.; Department of Clinical Health and Epidemiology (Stock), Dalhousie University, Halifax, NS; West Park Healthcare Centre (Cullen); Rehabilitation Sciences Institute (Cullen, Colantonio), University of Toronto; Institute for Clinical Evaluative Sciences (Colantonio), Toronto, Ont.

Contributors: Vincy Chan, David Stock, Nora Cullen and Angela Colantonio conceived and designed the study. David Stock formulated the methodology and analyzed the data. Vincy Chan and Binu Jacob drafted the manuscript. All of the authors contributed to data interpretation, critically revised the manuscript for important intellectual content, gave final approval of the version to be published and agreed to be accountable for all aspects of the work.

Funding: This project was funded by the Physicians' Services Incorporated Foundation and a Canadian Institutes of Health Research Project Grant.

Acknowledgement: The authors acknowledge Michelle Bartlett for her contributions in interpreting the findings for this study.

Disclaimer: This study was supported through provision of data by the Institute for Clinical Evaluative Sciences (ICES) and Cancer Care Ontario (CCO) and through funding support to ICES from an annual grant by the Ministry of Health and Long-Term Care and the Ontario Institute for Cancer Research (OICR). The opinions, results and conclusions reported in this paper are those of the authors and are independent from the funding sources. No endorsement by ICES, CCO, OICR or the Government of Ontario is intended or should be inferred. Parts of this material are based on data and information compiled and provided by the Canadian Institute for Health Information (CIHI). However, the analyses, conclusions, opinions and statements expressed herein are those of the authors and not necessarily those of CIHI.

Supplemental information: For reviewer comments and the original submission of this manuscript, please see www.cmajopen.ca/content/6/4/ E568/suppl/DC1. 